\title{
AN ALGORITHM FOR THE PATTERN COMPUTATION OF TRIANGULAR LATTICE
}

\author{
PHASED ARRAYS
}

\author{
S.A. Bokhari, N. Balakrishnan and P.R. Mahapatra \\ Department of Aerospace Engineering \\ Indian Institute of Science \\ Bangalore 560 012, India
}

\section{Introduction}

Numerical methods for array pattern optimization require efficient techniques for the pattern computation. Among the known geometries, the equilateral triangular lattice is a widely used configuration for large phased arrays in view of its substantial savings in hardware. Corey et al [1] have suggested use of Merserau's [2] hexagonal FFI algorithm for the efficient pattern computation. However, the coding of this algorithm is known to be rather complicated. Due to the large volume of literature on rectangular FFT algorithms, availability of computer programs, and the ease in coding some versions such as the row-column algorithm, it is advantageous to reduce the hexagonal FET to a rectangular FFT. O'neil et al [3] have developed an algorithm on these grounds. More recently, Geussom and Merserau [4] have shown that multi dimensional DFTs defined on arbitrary but periodic sampling lattices can be reduced to rectangular DFT's through a permutation of the input sequence.

In this paper, a hexagonal DFT convenient for triangular grid phased array computations is described. Based on the general theory [4], an algorithm for fast computation has been developed. Furthermore, an algorithm for carrying out the permutations of the input sequence "in place" has also been developed. This is of particular importance in dealing with FFTs of large order as it significantly reduces the auxilary storage required. The algorithm differs from that of o'neil et al [3] in that the periodicity lattices in both spatial and spatial frequency domains are identical. A main advantage of this algorithm is that it is remarkably simple to code and requires -about the same number of operations as the vector radix algorithm [2].

Application of the FFT algorithm for pattern computation often requires excessive "zero padding" to result in an adequate resolution in the spatial frequency domain. When computer storage becomes a limitation, some form of interpolation becomes necessary. An algorithm similar to the "pseudo sampling method" [5] has been described in this paper for use with the hexagonal FFT 


\section{The Hexagonal FFT algorithm}

While performing DFTs of a function of spatial coordinates, it is more convenient to adopt the definition given below instead of the more widely used definition in signal processing. This allows direct correspondance with the array factor. The hexagonal DFT of order $(\mathrm{N} \times \mathrm{N})$ for $\mathrm{N}=2^{\mathrm{m}}$, $\mathrm{m}$ being an integer, can be written as

$$
\begin{aligned}
& (\mathrm{N} / 2)-1 \quad(\mathrm{~N} / 2)-1 \\
& \mathrm{~F}_{\mathrm{h}}\left(\mathrm{k}_{1}, \mathrm{k}_{2}\right)=\Sigma \quad \Sigma \quad \mathrm{f}_{\mathrm{h}}\left(\mathrm{n}_{1}, \mathrm{n}_{2}\right) \exp \left[-j \pi\left(\left(2 n_{1}-n_{2}\right)\right.\right. \\
& n_{1}=-N / 2 \quad n_{2}=-N / 2 \\
& \left.\left.\cdot\left(2 \mathrm{k}_{1}-\mathrm{k}_{2}\right)+\mathrm{n}_{2} \mathrm{k}_{2}\right) / \mathrm{N}\right], \quad-\mathrm{N} / 2 \leq \mathrm{k}_{1}, \mathrm{k}_{2}<\mathrm{N} / 2
\end{aligned}
$$

where $f_{h}\left(n_{1}, n_{2}\right)$ denotes the array excitation coefficients with reference to the coordinate axes shown in Fig. $1, \mathrm{k}_{1}, \mathrm{k}_{2}$ denote the frequency variables in the spatial frequency domain and $\mathrm{F}_{\mathrm{h}}$ denotes the hexagonal DFT of $f_{h}$ with identical periodicity. The fundamental region of support in both domains is a rhombus and is convenient for storage as two dimensional arrays. Another advantage of this definition over the more general one [2] is that it reduces to a single square DFT. The inverse hex DFT is identical to Eqn. (1) except for a change in sign of the exponent, and a normalization factor $\left(1 / \mathrm{N}^{2}\right)$. The following definition of the square DFT has been employed since most available computer programs are based upon it.

$$
\begin{array}{cc}
F_{r}\left(k_{1}, k_{2}\right)=\sum & N-1 \\
n-1 & \left.n_{1}^{N} \quad f_{r}\left(n_{1}^{\prime}, n_{2}^{\prime}\right) \exp \left[-j 2 \pi(n+k\}+n_{2}^{\prime} k_{2}^{\prime}\right) / N\right] \\
& 0 \leq k_{1}^{\prime}, k_{2}<N
\end{array}
$$

Equation. (1) can be reduced to Egn. (2) by a nonlinear transformation of variables. The procedure with details omitted is given below.

(a) Form the perturbed array $f_{r}\left(n_{1}, n_{2}\right)=f_{h}\left(n_{1}, n_{2}\right)$

where $n_{1}=\left(n_{1}+n_{2}\right) \backslash N$ and $n_{2}=\left(n_{1}+2 n_{2}\right) \backslash N$

The modulo operation is denoted by ' $I$ ', and is assumed to wrap around and return a number between $-\mathrm{N} / 2$ and $\mathrm{N} / 2-1$.

(b) Compute the rectangular FFT $F_{r}\left(k_{j}, k_{2}\right)$ of $f_{r}\left(n_{j}, n_{2}\right)$

(c) Set $F_{h}\left(k_{1}, k_{2}\right)=F_{r}\left(k f\left|N, k_{2}\right| N\right)$ 
The computation in step (a) can be done "in-place" by exploiting the periodicity property of the indices generated by the application of the formula in (b). The input sequences can then be grouped into sets of different periodicities and the interchange of memory locations is then carried out. The algorithm, illustrated by the following four nested loops is valid for $N>2$.

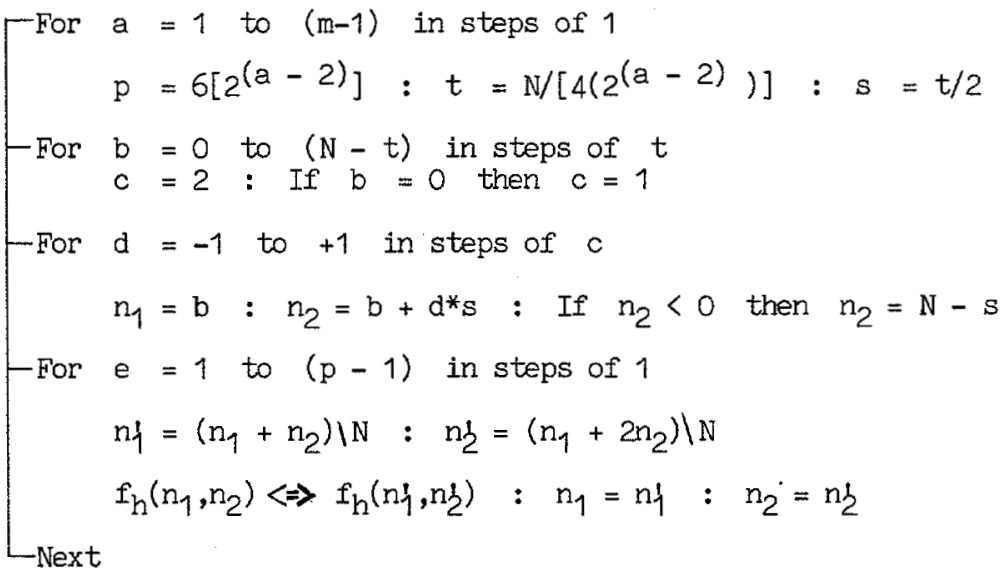

The symbol $\Leftrightarrow$ denotes an interchange of memory locations. The operation in step (c) can be done by a simple block interchange.

The algorithm for increasing the spatial frequency resolution consists of resampling the aperture distribution in steps of a chosen resolution factor, Fourier transforming the individual sets, phase compensation of each set followed by a summing operation.

\section{Numerical Illustration and Conclusion}

As an example, a 2437 element array located within a circle of radius $15 \lambda$ in the $Y-Z$ plane of the cartesian coordinate system, with $\Delta \mathrm{z}=0.5 \lambda$ is considered. The aperture distribution is taken as $\left[1-(\mathrm{r} / \mathrm{a})^{2}\right]^{2}$. The computed broadside pattern using a $64 \times 64$ point hex FFT with a resolution factor of 4 along both axes is shown in Fig. 2. The distorted appearance of the main beam is due to the rectangular plot of the hexagonal grid samples. Patterns at other angles can be readily obtained using the complex shifting theorem of DFTs.

The present algorithm provides a simplified, yet efficient method for the pattern computation of triangularly packed arrays. In situations where there is a storage limitation, the above algorithm combined with the hex FFT becomes convenient, however at the expense of computation time. The high resolution algorithm has the advantage of being exact and not involving the complexity of filter design as in other high resolution algorithms. 


\section{References}

[1] L.E. Corey, J.C. Weed and T.C. Speake, "Modeling of triangularly packed arrays using hexagonal FFT", IEEE AP-S Int. Symp. Digest, pp. 507-510, 1984.

[2] R.M. Merserau,"The processing of hexagonally sampled two dimensional signals", Proc. IEEE, vol. 67, pp. 930-949, 1979.

[3] D.R.O'neil, L.E. Corey and E.A. Nelson, "Evaluating the Fourier transform of a hexagonally sampled signal using rectangular DFT", Proc. IEEE South Eastcon, pp. 282-285, 1985.

[4] A. Guessom and R.M. Merserau,"Fast algorithm for the calculation of the multidimensional DFT", IEEE Trans Acoustics, Speech and Signal Processing, vol. ASSP-34, pp. 937-944, 1986.

[5] O.M. Bucci, G. D'Elia and G. Franceschetti, "Efficient computation of the far field of a parabolic reflector by pseudo sampling algorithm", IEEE Trans. Antennas Propagat., vol. AP-31, pp. 1259$1272,1983$.

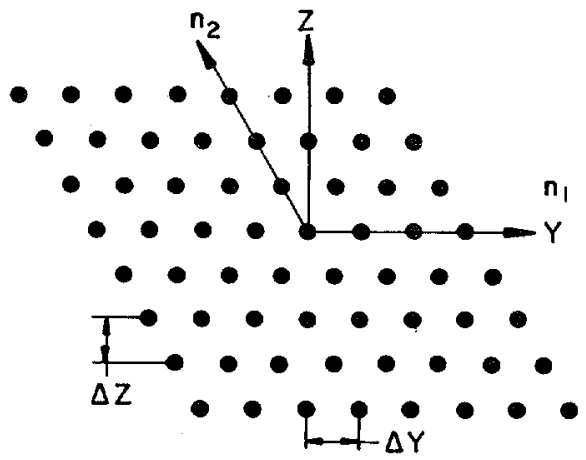

Fig.I Coordinate axes for the hexogonal geometry

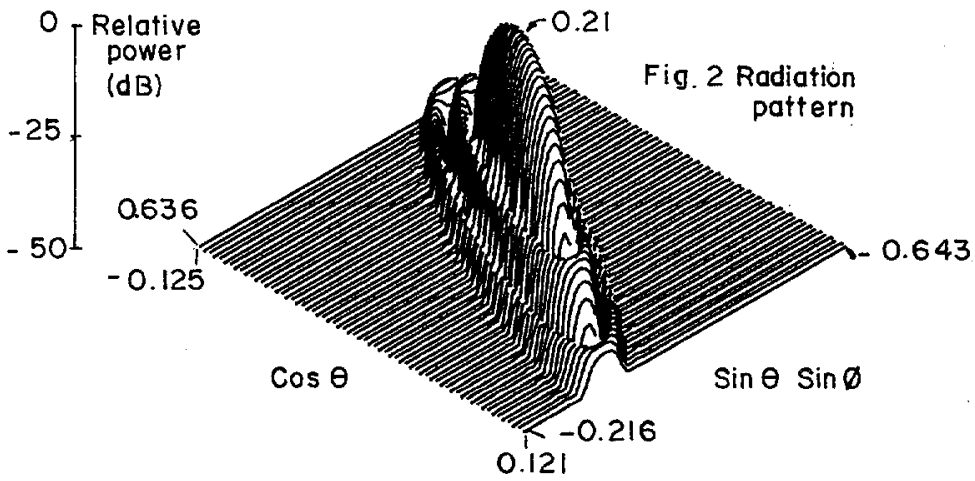

